\title{
Política de planeación de la educación superior en México e industria 4.0: 2013-2024
}

\author{
Higher education planning policy on México and industry 4.0: 2013-2024
}

\begin{tabular}{|c|c|}
\hline & Fernando, Cárdenas-Cabello ${ }^{2}$ \\
\hline & Centro Universitario CIFE, México \\
\hline (c) $(\$) \ominus$ & (iD https://orcid.org/0000-0003-1592-898X \\
\hline $\mathrm{BY}_{\mathrm{BY}} \mathrm{ND}$ & doi) $\mathrm{https}: / /$ doi.org/10.29105/pgc6.12-3 \\
\hline
\end{tabular}

\begin{abstract}
RESUMEN
Este trabajo se fijó por objetivo observar, medir y contrastar la evolución de la importancia que se ha dado a la formación para la industria 4.0 en la educación superior, por parte del gobierno federal de México en las dos más recientes administraciones, tomando como fundamento el documento básico de planeación de cada una de estas, el PND (Pan Nacional de Desarrollo) 2013-2018, y el PND 2019-2024. Para identificar cómo ha evolucionado la importancia de la formación para la industria 4.0 en la educación superior, en la política de planeación del gobierno federal de México. El acercamiento teórico se realizó desde la perspectiva de estudio de las políticas públicas, mientras que el método recurrido para el estudio del objeto en cuestión fue el análisis de contenido. Se identificó una cualitativamente mayor importancia prestada a la formación superior para la industria 4.0 en PND más reciente. Se concluye que la política de planeación federal mexicana -desde su documento básico- en materia de educación superior, se ha ido adecuando cada vez más a las nuevas realidades tecnológicas de la sociedad.
\end{abstract}

Palabras claves: Cambio tecnológico, desarrollo económico y social, enseñanza superior, política educacional, política gubernamental.

\section{ABSTRACT}

This work had the goal to observe, measure and contrast the evolution on importance given to formation for the 4.0 industry on higher education by Mexican federal government on the two most recent administrations, taking as basis the planning basic document from each, the NDP (National Development Plan) 2013-2018, and the NDP 2019-2023. To identify how it has changed the importance of formation for 4.0 industry in higher education, on the planning policy from Mexican federal government. The theoretical approaching was made through public policies study and the method used was content analysis. A more qualitatively important place was given to formation on higher education for the 4.0 industry in the most recent NDP. The conclusion is that Mexican federal planning policy -from its basic planning document- on higher education, has been adapting even more to technological new realities from the society

Keywords: Economic and social development, educational policy, government policy, higher education, technological change.

Recibido: 30 de Septiembre 2019 - Aceptado: 01 de Febrero 2020 - Corregido: 14 de Marzo 2020

Cómo referenciar este artículo:

Cárdenas-Cabello, F. (2020). Política de planeación de la educación superior en México e industria 4.0: 20132024. Politica Globalidad y Ciudadanía, 49-66. Recuperado de http://revpoliticas.uanl.mx/index.php/ RPGyC/article/view/146

1 Artículo de investigación derivado del proyecto de investigación: Educación superior e industria 4.0 en México, el cual finalizó en 2019

2 Doctor en Ciencias Políticas y Sociales por la Universidad Nacional Autónoma de México, Investigador del Centro Universitario CIFE y Profesor de la Universidad Autónoma del Estado de Morelos. Contacto: fernando.cardenas@uaem.mx 


\section{1.- INTRODUCCION}

La industria 4.0 es un concepto de gran actualidad y trascendencia, se refiere a la presente introducción de procesos informáticos en la producción y distribución de bienes, y en la provisión de servicios. Las instituciones de educación superior en México han comenzado una adaptación hacia esta tendencia actual de los procesos productivos en el mundo a través de la introducción de programas educativos que buscan formar profesionales acordes a esta nueva realidad de la industria 4.0.

El documento básico del gobierno federal en México, es el Plan Nacional de Desarrollo, es la carta de navegación en la que se encuentran las directrices para las diferentes acciones en los distintos sectores de la administración pública federal, y para el desarrollo de los varios programas sectoriales de las diversas secretarías que componen la estructura del gobierno; así como para el diseño de las políticas públicas, que deberán poner en marcha dichas dependencias.

La industria 4.0 es una noción que la academia alemana propuso al mundo desde el año 2011 para describir una realidad ya existente de la vinculación de los procesos productivos y comerciales con las tecnologías de la información vía remota, al ser un tema de central importancia en la actualidad, las directrices de la educación superior mexicana, tendrían que haberla considerado desde la elaboración del PND de la administración pública federal de México para el periodo 2013-2018, así como en el PND 2019-2024, es decir que los documentos mencionados debieron tener en cuenta la realidad de la transformación de los procesos productivos e informáticos, y la noción de la industria 4.0, para la elaboración de la planeación de la educación superior en México en los periodos referidos.

Como se planteó antes, este trabajo busca se exponer con qué amplitud e importancia se observa la planeación de políticas educativas federales para la industria 4.0, entre el sexenio actual y el inmediato anterior -con fundamento en el respectivo PND-, ello para identificar si ha habido una evolución en la preocupación por parte del gobierno federal por adecuar la educación superior en México a las necesidades de la realidad tecnológica de la sociedad actual.

\section{2.- FUNDAMENTO TEÓRICO}

\section{Políticas públicas}

El corpus teórico de las políticas públicas encuentra su origen en los planteamientos del politólogo norteamericano Harold Lasswell, quien en Las ciencias de política (The policy sciences), obra de 1951 sentó las bases para el análisis, diseño, implementación y evaluación de las acciones de gobierno, convertidas en programas, las políticas públicas; el enfoque de las ciencias de política quedó establecido de la siguiente manera por el propio Lasswell (Ed. 1996, p. 14, citado 
por Valencia y Álvarez, 2008, p. 99):

La orientación hacia las políticas tiene una doble dimensión: por una parte, se enfoca al proceso de la política y por la otra hacia las necesidades de inteligencia del proceso. La primera tarea busca desarrollar la ciencia de la formación y ejecución de las políticas, utilizando los métodos de investigación de las ciencias sociales y de la psicología. La segunda tarea busca mejorar el contenido concreto de la información y la interpretación disponible de los hacedores de las políticas y, por consiguiente, rebasa generalmente las fronteras de las ciencias sociales y de la psicología.

Evidentemente esta primera aproximación se complementa con el planteamiento de Muller (2007, Citado por Valencia y Álvarez, 2008, p. 96), para quien: “las políticas públicas son la forma como se le da coherencia, integralidad y sostenibilidad a las decisiones y acciones de los gobiernos". Al comentar que lo expuesto por Laswell se entiende en el ámbito de la acción del gobierno, el cual por un lado atiende a las funciones que le son ordinarias a través de acciones concretas, del mismo modo que adecua su operación ante demandas o "inputs" que la sociedad le va planteando, en función de problemáticas específicas nuevas o coyunturales, que se van incorporando a la agenda de lo público.

Una vez dicho que las políticas públicas son una de las actividades sustantivas del gobierno, es pertinente añadir un elemento importante presente en la definición propuesta por Evalsed (2008, Citado por Winchester, 2011, p. 5), quien afirma que estas son: "conjunto de actividades (programas, estrategias, procedimientos, leyes, reglamentos) dirigido hacia un objetivo general. Estas actividades frecuentemente se acumulan durante años". El elemento final de esta definición, no considerado hasta este momento, el temporal, es central al considerar que bajo la premisa del ensayo y error, las políticas públicas van enfocándose y mejorándose para atender de un modo más adecuado a los retos de la administración pública.

En un sentido amplio, las políticas públicas tienen por función, desde el punto de vista de Aguilar (1996, pp. 73-74):

atender problemáticas de auténtico interés general; facilitar el libre acceso de los ciudadanos interesados a la definición de los problemas y a la formulación de las políticas a través de canales abiertos, no selectivos, de opinión e influencia; construir y elegir políticas eficientes, maximizadoras de utilidades y minimizadoras de costos para el conjunto; corresponsabilizar a la ciudadanía en el tratamiento de sus problemas y satisfacción de demandas; introducir procedimientos de evaluación, crítica, responsabilización, corrección.

Las políticas públicas pues, son las acciones emprendidas por el gobierno para atender a las demandas permanentes de la población, así como a nuevos retos que se van presentando por la misma, y poseen un ciclo complejo, cuyos elementos mínimos son: formulación, implementa- 
ción y evaluación.

La elaboración de las políticas públicas en México a partir de la alternancia en el ejecutivo federal

En el año 2,000 el Partido Acción Nacional, resultó vencedor en las elecciones presidenciales en México, partido distinto al que había resultado ganador en los comicios para el mismo cargo por setentayún años, el Partido Revolucionario Institucional; este último había contado con el apoyo en el congreso hasta 1997 (Ver Cárdenas, 2020), año en que perdió la mayoría absoluta en la cámara de diputados, apoyo expresado especialmente en reformas legales, nombramientos y en aprobación de proyectos presupuestales.

A partir de la alternancia en el ejecutivo federal del año 2,000, el sistema político mexicano debió incluir de manera claramente decidida el elemento conciliador entre diversas fuerzas ideológico partidistas en la definición de políticas públicas, así como también la consideración de ciertos intereses y grupos de presión (especialmente empresariales), los cuales comenzaron a influenciar decisiones de política pública; sin ignorar otras voces que en oposición a esta liberalización de la sociedad fomentada desde el sistema político, pugnaban por la permanencia de un gobierno fuerte; y por supuesto, demandas sociales.

Entre las diversas fuerzas ideológicas encarnadas en el sistema de partidos mexicano a partir de la alternancia, se han encontrado expresiones que van desde el conservadurismo religioso, el liberalismo empresarial, el estatismo abierto a la promoción del libre mercado, los propulsores de la fortaleza del gobierno (incluso sus expresiones más agudas), hasta el ecologismo (que ha oscilado en alianza con un partido u otro sin clara definición).

Esta diversidad de perspectivas materializadas en intereses grupales y posturas partidistas, se ha considerado especialmente desde la alternancia en el ejecutivo federal, para la elaboración de las políticas públicas en México (Ver Béjar, 2018, y Medrano \& Muñoz, 2013) desde el año 2,000 hasta el periodo que cubre esta pesquisa, el 2019; la existencia de la diversidad de intereses partidista e ideológica, se encuentra presente, tanto en la propia política de planeación general expresada en su principal documento, el PND, como en las políticas públicas sectoriales.

En lo que respecta al PND 2013-2018, este muestra en su elaboración una tendencia al fortalecimiento del gobierno, con respecto de los grupos de poder presentes en el escenario del sistema político nacional, los cuales influyeron notablemente las decisiones de política pública en los dos sexenios previos, a partir de la alternancia; por su parte en el PND 2019-2024, esa misma tendencia de solidez gubernamental es más presente que en el mismo documento para el sexenio anterior, en consonancia con el discurso del partido que resultó ganador en las elecciones presidenciales de 2018.

Revista Política, Globalidad y Ciudadanía, Vol. 6. No. 12, Julio - Diciembre 2020, Universidad Autónoma de Nuevo León, Monterrey, México, ISSN 2395-8448. 49-66. http://revpoliticas.uanl.mx/index.php/RPGyC/article/view/146 


\section{La industria 4.0}

Es una noción de la mayor contemporaneidad, es un concepto acuñado primitivamente por los académicos alemanes hacia el inicio de la segunda década del siglo XXI en el escenario de la feria de Hannover de 2011; describe una realidad actual en la que los procesos informáticos están estrechamente vinculados con los procesos productivos y comerciales, es -en otras palabras- la cuarta revolución industrial o 4RI.

Para clarificar esta idea de la industria 4.0 y de la 4RI, es necesario recapitular en las anteriores revoluciones industriales, a saber:

La primera revolución industrial se gestó hacia la segunda mitad del siglo XVIII, con la introducción de las máquinas en los procesos de transformación, la invención de la máquina de vapor y del ferrocarril; y la utilización del insumo inicial para el funcionamiento de estos avances tecnológicos, el carbón.

La segunda revolución industrial se presentó a finales del siglo XIX, su principal característica es la recurrencia a la producción en serie en los procesos de manufactura, y la introducción de un nuevo elemento energético, la electricidad.

La tercera revolución industrial advino hacia la segunda mitad del siglo XX con la introducción de los ordenadores electrónicos y la presencia de un elemento popularizado en la última década del mismo siglo, la internet.

En cuanto a la cuarta revolución industrial, Schwab (2016, p. 13) dice:

Comenzó a principios de este siglo y se basa en la revolución digital. Se caracteriza por un internet más ubicuo y móvil, por sensores más pequeños y potentes que son cada vez más baratos, y por la inteligencia artificial y el aprendizaje de la máquina.

Sobre la misma revolución Fernández y Pajares afirman (Citados por Mendizábal, 2018, p. 3): "caracterizada por la digitalización del mundo industrial a través de los procesos de fabricación y interconexión de internet en las cosas”. Y continúa Schwab (2016, p. 13):

no solo consiste en máquinas y sistemas inteligentes y conectados. Su alcance es más amplio. Al mismo tiempo, se producen oleadas de más avances en ámbitos que van desde la secuenciación genética hasta la nanotecnología, y de las energías renovables a la computación cuántica.

Así, la industria 4.0, es la forma en que se desarrollan los procesos productivos y comerciales en el contexto de la 4RI y con los elementos de esta, como el IoT (internet de las cosas), y la ingeniería de punta aplicada en la biología, nanotecnología y energía.

Schwab denomina como megatendencias a ciertas condiciones o elementos tangibles que han posibilitado o favorecido las condiciones generales de la 4RI, las agrupa en tres tipos, físicas: vehículos autónomos, impresión 3D, robótica avanzada y nuevos materiales; digitales: como el IoT, sensores conectados en red, el blockchain (bitcoin) que modificará el sistema 
financiero internacional actual (Ver Lechuga, 2016), plataformas digitales (Uber, Airbnb); y biológicas: secuenciación genética, biología sintética, edición genética.

Como podemos observar, el concepto de industria 4.0 y la cuarta revolución industrial ${ }^{3}$ están absolutamente ligados:

El término industria 4.0 o también conocida como la cuarta revolución industrial o Fábrica Inteligente se empezó a utilizar en las industrias alemanas, misma que se define como un nuevo modelo de organización y de control de la cadena de valor a través del ciclo de vida del producto y a lo largo de los sistemas de fabricación apoyado y hecho posible por las tecnologías de la información. (Mendizábal y López, 2018, p 13).

La industria 4.0 y la 4RI, describen una condición actual, de interacción entre máquinas, sistemas informáticos, bases de datos y otros avances tecnológicos (materiales, energía, biotecnología, finanzas); capaces de vincularse sin una muy importante intervención del hombre que están transformando la manera en que se producen, transportan, almacenan y comercian bienes en las sociedades más tecnificadas, para modificar la estructura actual (Ver Lechuga, 2018) de la cadena productiva.

\section{La educación superior en México}

Al hablar de educación superior nos referimos invariablemente a aquella que se ofrece un nivel más alto a la de bachillerato (en países de habla española) o bachelor (en países de habla inglesa). En el informe final de la conferencia mundial sobre la educación superior, efectuada en París en 1998, se define así: "La educación superior es un componente de un sistema único que empieza con la educación para la primera infancia y la enseñanza primaria y continúa a lo largo de toda la vida" (UNESCO, 1998, p. 2). Cuya misión es: "educar, formar, llevar a cabo investigaciones y, en particular, contribuir al desarrollo sostenible y al mejoramiento del conjunto de la sociedad" (UNESCO, 1998 p. 2).

La Ley para la coordinación de la educación superior (1978, p. 1), de México establece en su artículo $3^{\circ}$ :

El tipo educativo superior es el que se imparte después del bachillerato o de su equivalente. Comprende la educación normal, la tecnológica y la universitaria e incluye carreras profesionales cortas y estudios encaminados a obtener los grados de licenciatura, maestría y doctorado, así como cursos de actualización y especialización.

3 No podemos ignorar la opinión de algunos autores como Brynjolfsson et al, para quien el estado actual de la tecnología no es una cuarta revolución industrial, sino la segunda era de las máquinas; la primera se caracterizó porque estas eran una herramienta para el hombre y era este quien tomada las decisiones que aquellas operaban, mientras que hoy las máquinas no requieren al ser humano para actuar. Brynjolfsson es citado por Blanco, Fondrona y Poveda (2017).

Revista Política, Globalidad y Ciudadanía, Vol. 6. No. 12, Julio - Diciembre 2020, Universidad Autónoma de Nuevo León, Monterrey, México, ISSN 2395-8448. 49-66. http://revpoliticas.uanl.mx/index.php/RPGyC/article/view/146 
En el caso de México, merece la pena distinguir en la educación superior ofrecida por el estado de manera pública, entre la de carácter universitario y la educación profesional técnica, modalidad promovida en México con mayor impulso ${ }^{4}$ a finales del siglo XX, con un ánimo más tendiente a la capacitación laboral, que a la formación integral que pretende ofrecer la universidad.

Para la Asociación Nacional de Universidades e Instituciones de Educación Superior (ANUIES, párrafos 4-6) de México:

La educación superior es el nivel con que culmina nuestro sistema educativo, lo cual implica que no debe ser considerado en forma aislada, sino en relación con los ciclos educativos que le preceden... las metas y objetivos que se propongan para mejorar las funciones de la educación superior, a nivel institucional, regional o nacional, formarán parte de la totalidad del sistema educativo nacional... la educación superior mexicana se realiza a través de instituciones que, en su conjunto, pueden clasificarse en públicas o privadas, en autónomas o estatales, en universidades o institutos tecnológicos o de diversa índole. Estas instituciones, aunque distintas por su régimen legal o por las áreas formativas a que se dedican, constituyen ante todo unidades sistémicas que, para preservar la cultura, formar profesionales en los diversos campos del saber, ejercitar la investigación, renovar el conocimiento y extender los beneficios de la cultura, emplean insumos y recursos, aplican procesos, y obtienen productos.

Las funciones básicas de la educación superior en México son la docencia, la investigación y la difusión de la cultura. Las áreas formativas de la educación superior en México son tres principalmente: la científica, la tecnológica y la humanística. En cuanto a sus objetivos "se identifican como objetivos, algunos aspectos que no se distinguen con claridad de las funciones básicas" (ANUIES, párr. 21) son los siguientes:

Formación de profesionales en los diversos campos del saber, la ciencia y la técnica, capaces de servir a su comunidad con eficiencia y responsabilidad;

Ejercicio de la investigación como tarea permanente de renovación del conocimiento y como una acción orientada a la solución, en diversos órdenes, de problemas nacionales, regionales y locales;

Extensión de los beneficios de la educación superior y de la cultura a todos los sectores de la comunidad, con propósitos de integración, superación y transformación de la sociedad. (ANUIES, párr. 22)

Las principales condiciones necesarias para el funcionamiento y la planeación del sistema

4 Pues la educación profesional técnica pública en México, inició formalmente en 1938 con la fundación del Instituto Politécnico Nacional.

Revista Política, Globalidad y Ciudadanía, Vol. 6. No. 12, Julio - Diciembre 2020 Universidad Autónoma de Nuevo León, Monterrey, México, ISSN 2395-8448. 49-66. http://revpoliticas.uanl.mx/index.php/RPGyC/article/view/146 
educativo superior de México son: estabilidad, desarrollo de la función crítica e incremento al financiamiento, a las que se añaden otras no esenciales pero de gran importancia: establecer acuerdos voluntarios y participativos de las instituciones; y creación de organismos, instancias y mecanismos requeridos para tomar decisiones.

Debe añadirse, que crónicamente la educación superior está presente en el documento básico que rige la administración pública sexenal mexicana, el Plan nacional de desarrollo (PND).

\section{El Plan Nacional de Desarrollo y la planeación de la educación superior en México}

La tradición de plasmar en un documento sexenal las directrices generales de actuación del gobierno federal de México se remonta a la administración 1934-1940, a cargo del general Lázaro Cárdenas (Ver PND, 2019-2024), desde entonces a la fecha todos los gobiernos federales mexicanos han elaborado un documento guía ${ }^{5}$ en el cual vierten directrices generales de acción en los distintos sectores de acción del gobierno.

Uno de estos sectores es precisamente el educativo, tema al cual básicamente todos -o por lo menos así es en los más recientes- los planes nacionales de desarrollo, le dedican un apartado importante. Martínez (2007) pone de manifiesto la desvinculación entre la política educativa federal plasmada en el PND, y los temas de interés y propuestas operativas del magisterio; supone que de considerarse estas, el logro de los objetivos educativos sería más eficiente.

El PND se elabora y publica para dar cumplimiento a lo estipulado en la Constitución Política de los Estados Unidos Mexicanos, específicamente en el artículo 25 inciso A; así como en los artículos 5to. Y 20mo. De la Ley de Planeación. Por su parte la planeación de la educación superior en México tiene sus antecedentes primitivos: en la primera reunión de rectores efectuada en 1940, en la creación de la Comisión Impulsora de la Investigación Científica y en la creación de la ANUIES en 1950. En cuya instauración estableció:

Responde a un supremo interés nacional la planeación de la enseñanza superior que debe concebirse en su proyección como un acto de autoridad de las instituciones educativas y su ejecución, por tanto, se fincará en convenios interuniversitarios dentro de un amplio plan de colaboración y reciprocidad (Citado por Rangel, p. 62).

En 1957, se dio otro paso en materia de planeación de la educación superior con la creación la Comisión de Estudios de Planeación Universitaria, mientras que para 1965 la Comisión Nacional de Planeación Integral de la Educación fue fundada. En 1978 se publicó el documento: La planeación de la educación superior en México, en el marco de la asamblea general de la

\footnotetext{
5 Es inevitable al hablar del Plan sexenal del gobierno de Lázaro Cárdenas del Río, remitirnos a los documentos programáticos de la URSS denominados plan quinquenal -los cuales probablemente influenciaron su creación- cuya finalidad era el desarrollo económico de esa nación y cuya primera versión se diseñó y aplicó a finales de la década de 1920.
} 
ANUIES del mismo año, en ese documento se expuso la necesidad de la existencia de un Plan Nacional de Educación Superior, el cual se materializó por primera vez en 1980.

La planeación es un aspecto fundamental en la política nacional, presente con claridad en uno de sus quehaceres fundamentales que es la educación, y en una de las partes integrantes de este sistema que es la educación superior.

\section{3.- MÉTODO}

\section{Diseño}

La metodología recurrida para el abordaje del objeto de estudio ha sido el análisis de contenido, la cual cuenta con una larga historia como herramienta de las ciencias sociales (Ver Andreu, 2011) y en cuyo desarrollo intervino de manera fundamental Harold Lasswell. El análisis de contenido puede ser definido según Berelson (1952, p. 18, citado por Andreu, 2011, p. 2) como: "una técnica de investigación para la descripción objetiva, sistemática y cuantitativa del contenido manifiesto de la comunicación”, por su parte Krippendorff (1990, p. 28, citado por Andreu 2011, p. 3) lo define así: "una técnica de investigación destinada a formular, a partir de ciertos datos, inferencias reproducibles y válidas que puedan aplicarse a su contexto".

Desde mediados del siglo pasado, los académicos e investigadores que se valían del análisis de contenido, debatieron sobre si privilegiar el aspecto cuantitativo o el cualitativo del mismo, especialmente por la introducción de programas informáticos, que permitían una contabilización detallada de palabras incluidas en los textos y discursos analizados. La perspectiva que ha sobresalido en este debate es la cualitativa, misma a la que se apega este estudio.

\section{Objeto o tema de análisis}

Como se expresó arriba esta investigación se propuso identificar la importancia que se ha dado en los Planes Nacionales de Desarrollo 3013-2018 y 2019-2024, a la formación para la industria 4.0 en la educación superior de México. Para identificar cómo ha evolucionado la importancia que ha ido adquiriendo la misma, y si esta presencia e importancia ha aumentado en el segundo documento con relación al primero.

\section{Participantes}

Los documentos analizados, fueron el PND 2013-2018, y el PND 2019-2024.

\section{Procedimiento}

La codificación consistió en que en cada documento se buscaron las alusiones a la educación superior, referidas a la formación para la industria 4.0; considerando como categorías de aná- 
lisis las mega tendencias de la 4RI propuestas por Schwab: físicas, digitales y biológicas. Es decir, se buscó el tratamiento que se dio en cada documento, a la formación de la educación suprior avocada a cada uno de estos aspectos de la industria 4.0.

\section{4.- RESULTADOS}

Políticas de educación superior para la industria 4.0 en el PND 2013-2018

El PND 2013-2018 está dividido en cinco grandes temas, a saber: paz, inclusión, educación, prosperidad y responsabilidad global; para cada uno de estos temas se especifican objetivos, estrategias y líneas de acción.

En lo respectivo al tema de educación superior e industria 4.0 es posible observar un objetivo exclusivo destinado a la innovación y la tecnología denominado: Hacer del desarrollo científico, tecnológico y la innovación pilares para el progreso económico y social sostenible. Previamente, en la descripción general y diagnóstico del tema III: México con educación de calidad, del cual se desprende el objetivo mencionado, se establece:

un México con Educación de Calidad propone implementar políticas de Estado que garanticen el derecho a la educación de calidad para todos, fortalezcan la articulación entre niveles educativos y los vinculen con el quehacer científico, el desarrollo tecnológico y el sector productivo, con el fin de generar un capital humano de calidad que detone la innovación nacional. (PND 2013-2018, p. 59).

El citado pasaje introduce la importancia de la vinculación entre el sector educativo y el científico, el tecnológico y el productivo, una de las tareas a las que debe atender la educación superior en general, en lo respectivo a las nuevas realidades a las que remite la noción de industria 4.0. En el mismo apartado introductorio sobre educación, observamos con claridad una alusión a esta misma realidad tecnológica, expresada del siguiente modo:

Las habilidades que se requieren para tener éxito en el mercado laboral han cambiado. La abundancia de información de fácil acceso que existe hoy en día, en parte gracias al Internet, requiere que los ciudadanos estén en condiciones de manejar e interpretar esa información. En específico, la juventud deberá poder responder a un nuevo ambiente laboral donde las oportunidades de trabajo no sólo se buscan, sino que en ocasiones se deben inventar ante las cambiantes circunstancias de los mercados laborales y la rápida transformación económica (PND 20132018, p. 60).

En este apartado podemos observar la clara referencia a las nuevas realidades de esta sociedad informatizada y los procesos económicos característicos de la misma, así como la preocupación por vincular a la juventud con estas nuevas realidades laborales por medio del fortalecimiento de la interrelación entre el sector productivo, la investigación y la educación. 
Más adelante en el mismo diagnóstico y presentación de este tema de educación, se platea también que las políticas y directrices del gobierno federal, en específico en lo relativo a la oferta educativa deben considerar las necesidades de la sociedad, así como también de la industria: "Para lograr una educación de calidad, se requiere que los planes y programas de estudio sean apropiados, por lo que resulta prioritario conciliar la oferta educativa con las necesidades sociales y los requerimientos del sector productivo". (PND 2013-2018, p. 62).

En la parte correspondiente a los objetivos, estrategias y líneas de acción sobre el rubro de educación, en el objetivo 3.1 denominado: Desarrollar el potencial humano de los mexicanos con educación de calidad; específicamente en la estrategia 3.1.3 denominada: Garantizar que los planes y programas de estudio sean pertinentes y contribuyan a que los estudiantes puedan avanzar exitosamente en su trayectoria educativa, al tiempo que desarrollen aprendizajes significativos y competencias que les sirvan a lo largo de la vida. Se establece de manera general para los distintos niveles educativos, la siguiente línea de acción: "Fomentar desde la educación básica los conocimientos, las habilidades y las aptitudes que estimulen la investigación y la innovación científica y tecnológica”. (PND 2013-2018, p. 124).

Más adelante en la siguiente estrategia, la 3.1.4 nombrada: Promover la incorporación de las nuevas tecnologías de la información y comunicación en el proceso de enseñanza aprendizaje. Se establece como línea de acción general para los distintos niveles educativos: "Intensificar el uso de herramientas de innovación tecnológica en todos los niveles del Sistema Educativo". (PND 2013-2018, p. 124). Identificamos por la disposición de esta estrategia, una preocupación en la planeación educativa federal del sexenio referido en los tres niveles, incluyendo el superior, una preocupación por el desarrollo de capacidades en el uso de los elementos tecnológicos, ya presentes en la vida cotidiana.

En el objetivo 3.2 denominado: Garantizar la inclusión y la equidad en el sistema educativo; específicamente en la línea de acción 3.2.3: Crear nuevos servicios educativos, ampliar los existentes y aprovechar la capacidad instalada de los planteles. Se establece la siguiente estrategia: "Fomentar la creación de nuevas opciones educativas, a la vanguardia del conocimiento científico y tecnológico" (PND 2013-2018, p. 126). Esta estrategia, habla de la necesidad de generar nuevas opciones formativas adecuadas a las tendencias de las nuevas tecnologías, como la informatización de los procesos productivos y comerciales de la industria 4.0.

Como se planteó al principio del estudio del documento PND 2013-2019, este incluye de manera específica un objetivo completo en el tema de educación destinado a la innovación y la tecnología, denominado: Hacer del desarrollo científico, tecnológico y la innovación pilares para el progreso económico y social sostenible. En la especificación de las estrategias para este objetivo se habla reiteradamente de la importancia de la tecnología, innovación e investigación 
60

y lo presente que deben tenerse todas estas en la creación de políticas públicas, pero es enfáticamente en la estrategia 3.5.4: Contribuir a la transferencia y aprovechamiento del conocimiento, vinculando a las instituciones de educación superior y los centros de investigación con los sectores público, social y privado. En la que se plasman líneas de acción específicas, relativas a la educación superior y las nuevas realidades tecnológicas e informáticas de la industria 4.0. Las cuales reproducen a continuación (PND 2013-2018, p. 129):

Apoyar los proyectos científicos y tecnológicos evaluados conforme a estándares internacionales.

Promover la vinculación entre las instituciones de educación superior y centros de investigación con los sectores público, social y privado.

Desarrollar programas específicos de fomento a la vinculación y la creación de unidades sustentables de vinculación y transferencia de conocimiento.

Promover el desarrollo emprendedor de las instituciones de educación superior y los centros de investigación, con el fin de fomentar la innovación tecnológica y el autoempleo entre los jóvenes.

Incentivar, impulsar y simplificar el registro de la propiedad intelectual entre las instituciones de educación superior, centros de investigación y la comunidad científica.

Propiciar la generación de pequeñas empresas de alta tecnología.

Impulsar el registro de patentes para incentivar la innovación.

Prácticamente todas la líneas de acción de esta estrategia se refieren a la disposición de políticas educativas de nivel superior para la investigación innovación y desarrollo tecnológicos, es decir, para la industria 4.0.

En la anterior revisión minuciosa del texto PND 2013-2019 podemos afirmar que existe en su desarrollo un tratamiento importante de las nuevas realidades tecnológicas actuales, valga decir de la cuarta revolución industrial (4RI) o industria 4.0, en la disposición de políticas públicas en materia de educación en general y educación superior en lo particular, especialmente porque el documento le dedica un objetivo completo del tema de educación a lo relativo al desarrollo tecnológico, la ciencia y la innovación.

\section{Políticas de educación superior para la industria 4.0 en el PND 2019-2024}

El documento PND 2019-2024 se compone de tres grandes ejes transversales agrupados en los siguientes temas: 1.-Igualdad de género, no discriminación e inclusión. 2.-Combate a la corrupción y mejora de la gestión pública. Y 3.-Territorio y desarrollo sostenible; contempla también tres ejes generales agrupados en los siguientes temas: 1.-Justicia y estado de derecho, 2.-Bienestar, y 3.-Desarrollo económico.

Revista Política, Globalidad y Ciudadanía, Vol. 6. No. 12, Julio - Diciembre 2020, Universidad Autónoma de Nuevo León, Monterrey, México, ISSN 2395-8448. 49-66. http://revpoliticas.uanl.mx/index.php/RPGyC/article/view/146 
A pesar de que en la disposición de los ejes principales del plan, no encontramos de manera explícita como uno de estos la educación, desde la introducción del documento podemos observar la alusión al fomento de las capacidades de las personas, trabajadores, gobierno, academia, industria y sociedad; para aprovechar los avances científicos, como se aprecia en el siguiente fragmento:

Es importante impulsar el desarrollo de nuevas capacidades de todas las personas para facilitar que la fuerza laboral, el gobierno y los sectores académico, productivo y social aprovechen las ventajas de estos cambios, promoviendo que el avance científico se traduzca en mayor bienestar. (PND 2019-2024, p. 13)

No obstante de que El PND 2019-2024, en su versión publicada por la cámara de diputados no establece de modo explícito en el índice los objetivos, indicadores y metas de trabajo. Sí se presentan en el cuerpo del texto objetivos que se ocupan de la educación. En el tema específico de Bienestar, en el objetivo 2.2, denominado de esta manera: Garantizar el derecho a la educación laica, gratuita, incluyente, pertinente y de calidad en todos los tipos, niveles y modalidades del Sistema Educativo Nacional y para todas las personas; en el desarrollo de este objetivo, podemos apreciar la importancia para la política educativa del actual gobierno sexenal, el desarrollo de capacidades en los sujetos en formación, para su adaptación a los cambios y condiciones tecnológicas actuales, como se observa en este texto:

El gran desafío de la educación en México es colocar a las niñas, niños, adolescentes y jóvenes en el centro de atención del Sistema Educativo Nacional, así como formarlos en el desarrollo de competencias que les permitan adaptarse a los cambios tecnológicos globales, a las nuevas exigencias del sector productivo y al auge del aprendizaje a través de plataformas virtuales. (PND 2019-2024, p. 93).

Al final de la redacción de este objetivo, se estipulan las estrategias específicas para su logro. Una de estas estrategias para alcanzar este objetivo, el 2.2, que si bien se refiere a la educación en todos los niveles, incluye por ello a la superior, delinea varias directrices de política pública del gobierno actual, entre las que se encuentra la que interesa a esta pesquisa, la formación para el uso de las nuevas tecnologías; la estrategia a la que nos referimos es la 2.2.3, cuya redacción es la siguiente:

Revisar los planes y programas de estudio en todos los tipos y niveles del Sistema Educativo Nacional, promoviendo la educación sostenible, artística, científica, tecnológica, financiera, ambiental, sexual, cívica, indígena, intercultural y comunitaria, que garanticen el derecho a la igualdad de género, la no discriminación y la eliminación de la violencia. (PND 2019-2024, p. 95).

Más adelante, ya en el eje general 3: Desarrollo económico, específicamente en el objetivo 
3.3, denominado: Promover la innovación, la competencia, la integración en las cadenas de valor y la generación de un mayor valor agregado en todos los sectores productivos bajo un enfoque de sostenibilidad. En la parte correspondiente a las estrategias para la logro del referido objetivo, entre otras, se establecen dos, que se relacionan con la vinculación entre el sector dedicado a la ciencia y los sectores de la sociedad para la producción de conocimiento y el desarrollo tecnológico. Así como el fomento al desarrollo de capacidades de las personas para la utilización de esas nuevas tecnologías en los sectores productivos, tales estrategias son:

3.3.1 Desarrollar el Sistema Nacional de Innovación de los sectores productivos para fortalecer la protección a los derechos de propiedad industrial e intelectual, que vincule a la comunidad científica con los sectores público, social y privado, a través de la generación de conocimiento de frontera y el desarrollo de tecnologías, para contribuir a la atención y solución de problemas nacionales prioritarios en materia social, económica y ambiental.

3.3.2 Impulsar el desarrollo y adopción de nuevas tecnologías en los sectores productivos y la formación de capacidades para aprovecharlas, vinculando la investigación con la industria y los usuarios y promoviendo métodos de producción sostenible y patrones de consumo responsable que promuevan el uso eficiente y racional del territorio y de sus recursos. (PND 2019-2024, p. 156).

En el mismo eje, tercero y último: Desarrollo económico, de manera concreta en el objetivo 3.5 intitulado: Establecer una política energética soberana, sostenible, baja en emisiones y eficiente para garantizar la accesibilidad, calidad y seguridad energética. Es posible identificar como directriz de política educativa, una estrategia, la 3.5.6, en la que se observa el impulso a uno de los temas de la 4RI, la generación de energía; y en este sentido se plantea la formación de personas dedicadas al uso y la investigación en este sector con altos estándares, como se aprecia a continuación:

Contribuir a la formación y fortalecimiento de capital humano y científico de alto nivel en el sector energético mexicano, de calidad mundial, tomando en cuenta su evolución y necesidades a largo plazo y bajo principios éticos, científicos y humanistas. (PND 2019-2024, p. 167).

Como se puede observar, a pesar de que el PND 2019-2024 no estipula de manera exclusiva en sus ejes transversales o generales, alguno dedicado de manera privativa al tema de educación, este es una preocupación permanente en diferentes apartados o ejes del documento, en los cuales se abordan de manera efectiva, diferentes planteamientos que muestran la voluntad de la administración federal mexicana 2018-2024, de atender desde el sector educativo, las presentes realidades tecnológicas y del conocimiento, relacionadas con el sector productivo; es decir, con la realidad de la 4RI o sociedad 4.0. 


\section{Discusión}

Los estudios actuales en México sobre planeación de la educación (Martínez, 2007) y planeación de la educación superior (Rangel, 2003); giran sobre su propio eje; del mismo modo lo hacen los trabajos que versan sobre educación superior e industria 4.0 (Escalante, 2019); no abundan, además del que se presenta aquí, trabajos que observen el cruce entre la planeación de la educación superior y la industria 4.0. Esta es una veta de reflexión que podrá proveer de propuestas de mejoramiento de la planeación de la educación superior dirigida a esta realidad, cada vez más presente en la vida de las personas, la industria 4.0, ó 4RI.

\section{5.- CONCLUSIONES}

La contrastación del contenido y la manera de expresarlo entre los documentos rectores de las dos administraciones federales mexicanas analizados, requiere el presentar las conclusiones en dos niveles, el primero de forma, sobre lo expuesto con relación a la educación superior para la industria 4.0, y el segundo de fondo, sobre el contenido y presentación general de la información vertida en cada una de las dos versiones revisadas del PND.

Conclusiones de forma: Tanto en el documento PND 2013-2018, como en el PND 20192024, está presente la preocupación por formar recursos humanos a nivel profesional que atiendan a las realidades de la industria 4.0.

En cuanto a las unidades de registro -las denominadas mega tendencias por Schwab-: físicas, digitales y biológicas, ambos documentos se preocupan de su importancia en la formación de la educación a nivel superior para las digitales, aunque ninguno de los dos habla con profundidad sobre las biológicas, y en cuanto a las físicas, sólo el PND 2019-2024 se refiere con claridad al tema de formación para la generación de nuevas alternativas de energía.

Conclusiones de fondo: en primer lugar, desde la portada, el título del PND 2019-2024, se escribe no sólo en castellano sino también en idiomas y lenguas autóctonas de México, el lenguaje de género es más inclusivo y se evitan los sustantivos en masculino.

Por su parte el PND 2013-2018 tiene la virtud de presentar desde el índice de manera desplegada, además de los grandes temas o ejes, los objetivos; mientras que en el 2019-2024, sólo se aprecian los ejes o grandes temas; la presentación de los objetivos en el índice, permite una mayor claridad en la comprensión del documento y un mejor manejo del mismo.

En el PND 2019-2024, los indicadores de los objetivos se expresan en términos numéricos, con lo cual su evaluación será más fácilmente ponderable, y se podrá verificar al final de la administración si el objetivo se cumplió o no. Se observa que al final de cada eje general en este documento se incluye un listado de propuestas ciudadanas para el gobierno.

Por último, es notorio el siguiente aspecto, el PND 2013-2018 incluyó al inicio del docu- 
mento una imagen del respectivo presidente de la república, mientras que en el 2019-2024 no se aprecia la imagen del presidente en turno.

Como conclusión final: cuantitativamente se presenta un mayor contenido sobre la importancia de la educación superior para la industria 4.0 en el PND 2013-2018, aunque se observa una mayor preocupación en términos cualitativos por la formación en la educación superior de México para la industria 4.0 en el PND 2019-2024. Esta aseveración se deriva de que de las 3 mega tendencias de la 4RI, que para fines metodológicos de la presente pesquisa se convirtieron en categorías de análisis (físicas, digitales y biológicas), el segundo documento mencionado (2019-2024) se ocupa de la formación en la educación superior enfocada a atender a dos de esos tres temas, mientras que el otro (2013-2018) sólo contempla uno de los tres. Así las cosas, la política de planeación federal de México en lo que respecta a la importancia que presta a la educación superior para la industria 4.0, ha evolucionado preocupándose cada vez más por atender a estas realidades en aumento de la sociedad actual.

\section{REFERENCIAS}

CAguilar, L. (1996). El estudio de las políticas públicas. México D.F.: Miguel Ángel Porrúa. Andreu, J. (2011). Las técnicas de análisis de contenido: Una revisión actualizada, pp. 1-34. Recuperado de http://mastor.cl/blog/wp-content/uploads/2018/02/Andreu.-analisis-decontenido.-34-pags-pdf.pdf

ANUIES México (s.f.). La educación superior en México. Recuperado de http://publicaciones. anuies.mx/acervo/revsup/res029/txt1c.htm\#top

Béjar, L. (2018). Partidos políticos y políticas públicas ¿Qué pasa con la representación política en México? Estudios políticos. (44), 41-64. Recuperado de: http://www.scielo.org.mx/pdf/ ep/n44/0185-1616-ep-44-41.pdf

Blanco, R.; Fontdrona, J. y Poveda, C. (2017). La industria 4.0: El estado de la cuestión. Recuperado de Www.mincotur.gob.es/Publicaciones/Publicacionesperiodicas/ EconomiaIndustrial/RevistaEconomiaIndustrial/406/BLANCO,\%20FONTRODONA\%20 Y\%20POVEDA.pdf

Cárdenas, F. (2020). La relación ejecutivo legislativo en México en: Reconfiguración de la relación entre los poderes ejecutivo y legislativo en Morelos a partir de la alternancia: 2000-2018. Manuscrito no publicado.

Escalante. A. (2019). El reto de la educación superior ante la industria 4.0. Recuperado de: https://archivos.juridicas.unam.mx/www/bjv/libros/12/5645/19.pdf 
Lasswell, H. (1996). La concepción emergente de las ciencias de política. En: L. F. Aguilar Villanueva, El estudio de las políticas públicas. México D.F.: Miguel Ángel Porrúa.

Lechuga, J. (2016). Módulo de Finanzas Internacionales. Barranquilla: Ediciones Instituto Tecnológico de Soledad Atlántico.

Lechuga, J. (2018). Wood industry in Colombia: key resources for export performance. Revista Academia \& Negocios RAN, 4 (1), 15-23.

Ley para la coordinación de la educación superior. (1978). México. Recuperado de: https://www. sep.gob.mx/work/models/sep1/Resource/558c2c24-0b12-4676-ad90-8ab78086b184/ley_ coord_educ_superior.pdf

Martínez, H. (2007). Comparativo entre el Plan Nacional de Desarrollo y las conclusiones y propuestas del IV Congreso Nacional de Educación y del Segundo Encuentro Nacional de Padres de Familia y Maestros. Revista latinoamericana de estudios educativos, 36, 49-72. Recuperado de: https://www.redalyc.org/pdf/270/27037203.pdf

Medrano, R., y Muñoz,A. (2013). Partidos políticos y formulación de políticas: Las percepciones de la militancia en México. Espacios públicos, 16 (38), 23-40. Recuperado de: https:// www.redalyc.org/pdf/676/67629717003.pdf

Mendizábal, G. (2018). Seguridad social y la industria 4.0. Recuperado de https://archivos. juridicas.unam.mx/www/bjv/libros/12/5645/4.pdf .

Mendizábal, G. y López, E. (2018). ¿Nuevo modelo de seguridad social en la industria 4.0?, Revista internacional y comparada de relaciones laborales y derecho del empleo, 6, 298327.

Plan Nacional de Desarrollo 2013-2018. Recuperado de https://www.snieg.mx/contenidos/ espanol/normatividad/MarcoJuridico/PND_2013-2018.pdf

Plan Nacional de Desarrollo 2019-2024. Recuperado de http:/gaceta.diputados.gob.mx/ PDF/64/2019/abr/20190430-XVIII-1.pdf

Rangel, A. (2003). La planeación de la educación superior en México, OMNIA, 19, pp. 69-70, recuperado de http://www.posgrado.unam.mx/publicaciones/ant_omnia/Esp_16/11.pdf

Schwab, K. (2016). La cuarta revolución industrial. Barcelona (España): Editorial Debate.

UNESCO. (1998). La educación superior en el siglo XXI visión y acción, París. Recuperado de https://unesdoc.unesco.org/ark:/48223/pf0000116345_spa 
66

Valencia, G. y Álvarez, Y. (2008). La ciencia política y las políticas públicas: notas para una reconstrucción histórica de su relación. Estudios politicos, 33, 93-121. Recuperado de https://dialnet.unirioja.es/descarga/articulo/5263710.pdf

Winchester, L. (noviembre de 2011). La formulación e implementación de las políticas públicas en ALC. Curso Políticas presupuestarias y gestión por resultados en América Latina y el Caribe, Santiago de Chile. Recuperado de https:/www.cepal.org/ilpes/noticias/ paginas/4/45114/Pol\%C3\%ADticaspublicasenALC_Winchester.pdf 